\title{
Infection Dynamics Vary between Symbiodinium Types and Cell Surface Treatments during Establishment of Endosymbiosis with Coral Larvae
}

\section{Line Kolind Bay ${ }^{1,2, *}$, Vivian Ruth Cumbo ${ }^{2,3,4}$, David Abrego ${ }^{1}$, Johnathan Travis Kool ${ }^{1,2}$, Tracy Danielle Ainsworth ${ }^{2}$ and Bette Lynn Willis ${ }^{2,3}$}

1 Australian Institute of Marine Science, PMB \#3, Townsville MC, Queensland 4810, Australia; E-Mails: d.abrego@aims.gov.au (D.A.); johnathan.kool@jcu.edu.au (J.T.K.)

2 ARC Centre of Excellence for Coral Reef Studies, Townsville, Queensland 4811, Australia; E-Mails: vcumbo@csun.edu (V.R.C.); tracy.ainsworth@jcu.edu.au (T.A.); bette.willis@jcu.edu.au (B.L.W.)

3 School of Marine and Tropical Biology, James Cook University, Townsville, Queensland 4811, Australia

4 James Cook University, Townsville, Queensland 4810, Australia

* Author to whom correspondence should be addressed; E-Mail: 1.bay@aims.gov.au; Tel.: +61-7-47-534179; Fax: +61-7-4772-5852.

Received: 12 July 2011 / Accepted: 18 July 2011 / Published: 19 July 2011

\begin{abstract}
Symbioses between microbes and higher organisms underpin high diversity in many ecosystems, including coral reefs, however mechanisms underlying the early establishment of symbioses remain unclear. Here we examine the roles of Symbiodinium type and cell surface recognition in the establishment of algal endosymbiosis in the reef-building coral, Acropora tenuis. We found 20-70\% higher infection success (proportion of larvae infected) and five-fold higher Symbiodinium abundance in larvae exposed to ITS-1 type C1 compared to ITS- 1 type D in the first $96 \mathrm{~h}$ following exposure. The highest abundance of Symbiodinium within larvae occurred when C1-type cells were treated with enzymes that modified the $40-100 \mathrm{kD}$ glycome, including glycoproteins and long chain starch residues. Our finding of declining densities of Symbiodinium C1 through time in the presence of intact cell surface molecules supports a role for cell surface recognition molecules in controlling post-phagocytosis processes, leading to rejection of some Symbiodinium types in early ontogeny. Reductions in the densities of unmodified C1 symbionts after $96 \mathrm{~h}$, in contrast to increases in D symbionts may suggest the early
\end{abstract}


initiation of a winnowing process contributing to the establishment of Symbiodinium D as the dominant type in one-month old juveniles of $A$. tenuis.

Keywords: symbiosis; coral; Symbiodinium; Acropora tenuis; glycan; lectin; glycome; winnowing; symbiont dynamics; host selection

\section{Introduction}

Symbioses between higher organisms and microbes are widespread in the animal and plant kingdoms and often underpin high biodiversity (reviewed in [1,2]). Symbiotically derived benefits for hosts may be nutritional, for example, up to $90 \%$ of plants form associations with arbuscular mycorrhizal (AM) fungi that fix nitrogen essential for plant growth [3]. Similarly, hermatypic corals routinely obtain $>90 \%$ of their energy requirements from their photosynthetic endosymbionts [4]. Symbiotic relationships may confer other physiological advantages to hosts, such as enhanced thermal and drought tolerance in plants when associated with AM fungi and viruses [5]. The coral-Symbiodinium association also affects the growth, survival, nutritional status and bleaching tolerance of coral hosts [6-10]. In light of these far reaching benefits, the break-up of symbiotic associations can have widespread ecosystem implications, particularly when hosts are the source of resources and habitat structural complexity supporting associated organisms, as is the case with trees and corals. Many symbioses are particularly sensitive to fluctuations in ambient environmental conditions, and it is widely recognised that coral bleaching, the process by which coral hosts and Symbiodinium populations disassociate during stress, is a major threat to coral reefs world-wide [11]. As environmental fluctuations associated with human activities continue to increase, there is an urgent need to better understand symbiotic interactions in many systems [12].

The processes by which symbioses are established can affect host symbiont diversity and in turn, their ecology and evolution [1]. In symbioses with vertical transmission, symbionts are passed from parents to offspring; hence, their genetic and physiological diversity within offspring will be determined predominantly by the composition and availability of symbionts in the parental pool [1]. In symbioses with horizontal transmission, symbionts are procured exclusively from the environment through a colonisation process we refer to here as "infection", without implying negative aspects of the term associated with pathogen interactions [1,2]. Horizontal transmission is the dominant process of Symbiodinium infection in scleractinian corals $[13,14]$ and may provide corals with flexibility to associate with a range of symbiont genotypes and the opportunity for procurement or maintenance of optimal symbiont types under the prevailing environmental conditions [12]. For example, some corals may host multiple symbiont types [15] and may become dominated by more tolerant ones following environmental perturbations $[8,16]$. Despite this flexibility, many horizontally transmitted symbioses are specific: corals associate with a single Symbiodinium type or a small subset of available types and revert to pre-stress Symbiodinium complements relatively quickly following stress-induced shuffling of dominant symbiont types [16]. To understand the mechanisms that allow this specificity to occur, it is necessary to identify the cellular mechanisms underlying the initiation and maintenance of symbiosis in different coral species. 
Hypotheses about the cellular mechanisms that underlie coral symbioses can be based on knowledge from other cnidarian symbioses, including symbioses between Hydra viridis and Chlorella [17,18], Cassiopeia xamachana and Symbiodinium spp. [19-22], as well as those involving anemones and corals [23]. These studies demonstrate that the complexity of cellular and molecular mechanisms that underpin successful cnidarian symbioses include both differences in initial infectivity of symbionts [17,23,24] and post-infection re-sorting of initial symbiont complements [17,23,25]. The molecular and cellular communication mechanisms underlying the establishment, reshaping and maintenance of cnidarian symbioses involve a range of cellular exudates and surface molecules [26] combined described by the glycome [27]. Long chain starch residues and glycoproteins are part of the Symbiodinium glycome in culture and in hospite [20,28,29], suggesting that they may play a role in cell recognition and/or host nutrition [19,20]. Cassiopea xamachana antibodies recognised exudates from Symbiodinium types capable of inducing developmental metamorphosis, but did not recognise those from other Symbiodinium types that do not induce metamorphosis. This supports a signalling function for Symbiodinium glycomes in this species [20]. Molecules found on the surfaces of host and symbiont cells, like glycans and lectins, have also been implicated in cellular communication in cnidarian symbioses [29-32]. Lectins are carbohydrate-binding proteins found on host cells that conjugate with mono- or simple oligosaccharides [33]. The glycomes of phytoplankton, including Symbiodinium spp., contain a range of oligosaccharide molecules that vary within and among species [29-31,34,35]. In particular, glycans containing mannose residues and $N$-acetyl groups have been detected on the surface of a broad range of Symbiodinium types [29]. Cell surface sugars can be experimentally modified using either enzymes that cleave terminal residues or artificial lectins that block receptor sites. Experimental removal of specific cell surface cues enables their importance to be examined under controlled symbiont densities and environmental conditions. For example, aposymbiotic Fungia scutaria larvae and bleached Aiptasia pulchella adults showed significantly lower uptake of most enzyme- or lectin-treated Symbiodinium cells compared to unmodified Symbiodinium, despite cells representing the dominant symbiont type found in adults of host species [30,31]. This suggests that $F$. scutaria and $A$. pulchella use cell-surface sugars as cues for the procurement of symbiont types that are dominant in adult hosts. The role of cell surface recognition in the onset of other cnidarian symbioses, particularly those where hosts associate with multiple symbiont types during early ontogeny [7,36], is currently not known.

Coral endosymbionts of the genus Symbiodinium are genetically diverse and nine main clades (A-I) are currently recognized, each containing many sub-clades or types [37-40]. Although the distribution and abundance of free-living symbionts are generally unknown for most types and locations, Symbiodinium cell densities can be high in sediments on some reefs [41] and higher initial uptake by larvae occurs when exposed to reef sediments compared to water column samples [42]. The larvae and juveniles of many coral species are able to take up a range of Symbiodinium types $[36,43]$ that can differ from those dominating adult corals [7,25,36,44-46]. Relative infection rates can differ among symbiont types and can be higher with types that dominate in adult symbiosis [23,24,46] but see [43]. Ontogenetic shifts in dominant symbiont types occur in some corals, with adults dominated by different symbiont types than juveniles at the same reef locations [7,25,36,47]. For example, adult colonies of Acropora tenuis associate with $\mathrm{C} 1$ and $\mathrm{C} 2$ Symbiodinium throughout their Indo-Pacific range $[48,49]$, with adult $A$. tenuis associating almost exclusively with $\mathrm{C} 1$ on Magnetic Island reefs in 
the central region on the Great Barrier Reef [50,51]. In contrast, juveniles (1 month-2 years) are dominated by type D symbionts on Magnetic Island reefs [7,47]. Such temporal patterns in coral-Symbiodinium associations are consistent with a number of alternative interpretations, including differences in initial infection rates among symbiont types, differences in post-infection survival and growth rates among symbiont types, the presence of a host-mediated, post-infection "winnowing" mechanism [2], and/or differences in the abundance of Symbiodinium types among reefs, with one or all of these processes shaping specificity of symbiosis during early ontogeny. The relative importance of host recognition mechanisms, symbiont availability and/or post-infection symbiont interactions in this winnowing process is currently unclear.

Here we examine the proportion of larvae infected with Symbiodinium cells and symbiont abundance over four days in $A$. tenuis larvae provided with controlled quantities of $\mathrm{C} 1$ and $\mathrm{D}$ Symbiodinium (sensu [51]). We examine the roles of cell surface molecules on the early stages of symbiosis by comparing uptake and proliferation of untreated positive control cells versus cells whose surface had been modified using either enzymes to denaturate glycoproteins or lectins to mask glycans. We found significantly more larvae were infected with more C1-type symbionts compared to D. We observed the highest uptake of C1 Symbiodinium when cell surface glycome was modified using enzymes, suggesting a role of glycoproteins and long chain starch residues in post-phagocytosis processes rather than initial uptake, during the early establishment of this coral-Symbiodinium symbiosis. We also report evidence of the initiation of a possible winnowing process to establish the dominant juvenile symbiont type after 5 days.

\section{Experimental Section}

\subsection{Larval Husbandry}

Fecund colonies of Acropora tenuis were collected from Magnetic Island, Australia (19 $10^{\circ} \mathrm{S}$, $146^{\circ} 50^{\prime} \mathrm{E}$ ) and spawned at 7:00 pm on the 20th of October 2008, 5 days after the full moon. The colonies were kept in individual $60 \mathrm{~L}$ tanks with flow-through $1 \mu \mathrm{m}$ filtered seawater (FSW). Gametes were collected from spawning colonies and fertilized in separate containers filled with $1 \mu \mathrm{m}$ FSW. After fertilization, the embryos were transferred to $600 \mathrm{~L}$ tanks with flow-through FSW, where they were raised until they developed into planula larvae. Four days after spawning, the larvae were transferred to a temperature-controlled laboratory $\left(27^{\circ} \mathrm{C}\right)$ at the Australian Institute of Marine Science for allocation into experimental treatments. Planula larvae were eight days old (12 days post fertilisation) when the experiment commenced.

\subsection{Preparation of Freshly Extracted Symbiodinium Cells}

Fresh extracts of Symbiodinium ITS-1 types D and C1 were obtained from fragments of Acropora millepora and A. tenuis colonies, respectively, collected from Magnetic Island (19¹0'S, $\left.146^{\circ} 50^{\prime} \mathrm{E}\right)$. Coral tissue containing Symbiodinium cells was isolated by high-pressure airbrushing and collected in a bag containing $0.2 \mu \mathrm{m}$ FSW. Slurries were homogenized and filtered through a $60 \mu \mathrm{m}$ mesh, centrifuged (3000 g for $4 \mathrm{~min}$ ) and washed 3 times, then filtered through a $10 \mu \mathrm{m}$ membrane (Millepore) to remove most coral cells and skeletal debris. Symbiodinium extracts were kept in $0.2 \mu \mathrm{m}$ 
FSW for $26 \mathrm{~h}$, after which cell densities were determined in five replicate samples per type using a haemocytometer. DNA was extracted from donor coral branches and Symbiodinium cell extracts, and the dominant Symbiodinium type was confirmed using Single Strand Conformation Polymorphism (SSCP) [52]. To examine differences in cell integrity of Symbiodinium C1 and D, the proportion of dead cells was quantified in fresh Symbiodinium isolates in Oct 2010. Symbiodinium C1 cells were extracted from A. tenuis and D cells from Acropora pulchra and genotyped as above. A. millepora could not be used as the D-type donor because of significant mortality at the only known location where this species associates almost exclusively with D-type Symbiodinium [36]. Dead cells were stained with Evans blue [53] immediately following extraction, and at 24, 48 and $72 \mathrm{~h}$ after extraction. Four replicate counts of the number of stained dead cells vs. unstained live cells were conducted using a haemocytometer and light microscope.

\subsection{Cell Surface Modifications of Freshly Isolated Symbiodinium Spp.}

Five symbiont cell surface modifications were undertaken using two enzymes [ $\alpha$-Amylase (Sigma A6255) and trypsin (Sigma T6567)] and three lectins [(Concanavilin A (ConA) from Jack bean (Sigma C7275), LPA from Limulus polyphemus (Sigma L2263) and WGA from Triticum vulgaris (Sigma L9640)]. The two enzyme treatments modified molecules on the surface of Symbiodinium cells: $\alpha$-Amylase hydrolyses $\alpha-(1,4)$ glycan linkages, whereas trypsin hydrolyses any exposed peptide bonds on the carboxyl side of arginine and lysine residues. Specifically, trypsin digests the $40-100 \mathrm{kD}$ range of glyco-protein exudates of Symbiodinium microadriaticum [20]. The lectin treatments blocked specific glycans: ConA blocked $\alpha$-mannose and $\alpha$-glycose residues; LPA blocked $N$-acetyl neuraminic acid, glycuronic acid and phosphorylcholine analog residues; and WGA blocked $N$-acetyl- $\beta$-D-glycosaminyl and $N$-acetyl- $\beta$-D-glycosamine oligomers. The lectin and enzyme treatments were selected to overlap with those used in previous studies (as outlined in [30,31]) and to reflect the likely surface glycans of Symbiodinium strains used [29]. Symbiont cells were diluted to $1 \times 10^{5}$ cells $/ \mathrm{mL}$ and digested with final concentrations of either $5 \mathrm{mg} / \mathrm{mL} \alpha$-Amylase (resuspended in $25 \mathrm{mM}$ Tris- $\mathrm{HCl} \mathrm{pH} 7.5,100 \mathrm{mM} \mathrm{KCl}$ ), $6 \mu \mathrm{g} / \mathrm{mL}$ trypsin (resuspended in $1 \mathrm{mM} \mathrm{HCl}$ ) or $0.1 \mathrm{mg} / \mathrm{mL}$ of lectin (resuspended in PBS) (as outlined in [30,31]). Cells in all treatments were incubated at room temperature for $2 \mathrm{~h}$ in the dark, mixing gently every $20 \mathrm{~min}$. Following incubation, algae were centrifuged at $3000 \mathrm{~g}$ for $5 \mathrm{~min}$, washed 3 times and re-suspended in fresh $0.2 \mu \mathrm{m} \mathrm{FSW}$ before infection.

To examine the binding of lectins to the surface of Symbiodinium cells, Alexa488 fluoro-labelled ConA and WGA lectins (Invitrogen: C11252 and W11261) were hybridised to fresh extractions of Symbiodinium C1 and D obtained in Oct 2010, as described above. Alexa488 labelled LPA was not available and therefore could not be tested. For each cell type, approx $1 \times 10^{7}$ cells were preserved in $4 \%$ paraformaldehyde $24 \mathrm{~h}$ after extraction and stored at $4{ }^{\circ} \mathrm{C}$. After 4 days, fixed cells were spun at $3000 \mathrm{~g}$ for $6 \mathrm{~min}$ and washed twice in PBS (pH 7.2). Separate aliquots of $500 \mu \mathrm{L}$ $\left(1 \times 10^{6}\right.$ Symbiodinium cells $\left./ \mathrm{mL}\right)$ were incubated with the two fluorescently labelled lectins in a final concentration of $50 \mu \mathrm{g} / \mathrm{mL}$ for one hour in the dark, alongside a non-modified control. In two separate $500 \mu \mathrm{L}$ aliquots, C1- and D-type cells were incubated with ConA and WGA lectins that had been pre-exposed to lectin inhibitors (WGA with $N$-acetyl-D-glucosamine [Sigma A8625]; ConA with 
Methyl alpha-D-mannopyranoside [Sigma: M6882]) at a concentration of $1 \mathrm{M}$ for $1 \mathrm{~h}$. Following incubation, cells were washed twice in PBS and resuspended in $100 \mu \mathrm{L}$ PBS. Ten microlitres of each cell suspension was placed into 4 wells of a teflon coated eight-well microscope slide (ProSciTech G350805-BK) and mounted with fluorescent mounting media (VectaSheild H-1400) for imaging using confocal microscopy. Unstained control cell suspensions were also prepared for both C1 and D type symbionts to allow for spectral profiling of the innate fluorescence. Symbiont cells were imaged using a Zeiss Meta 710 confocal microscope, excited using a $488 \mathrm{~nm}$ (argon) laser. Emissions were collected using 34 spectral detection channels within 488 to $690 \mathrm{~nm}$ emission. Following spectral mapping of both innate fluorescence and labelled cell fluorescence, online profiling was used to image 800-1100 individual cells of both labelled and control cell suspensions. The use of online fingerprinting and confocal microscopy to determine the proportion of cells labelled allows for the resolution of multiple distinct fluorescent emissions associated with a single cell within a pixel resolution [54]. The auto-fluorescence of the $5 \mu \mathrm{m}$ diameter accumulation body within the $10 \mu \mathrm{m}$ Symbiodinium cell results in a similar $590 \mathrm{~nm}$ emission peak as the Alexa488 surface labelled lectins. Therefore this method allows for high-resolution spectral separation of labelled and unlabelled cells, which is otherwise unachievable using flow cytometry.

\subsection{Experimental Design}

Approximately 200 larvae were placed in experimental tubs containing $200 \mathrm{~mL}$ of $0.2 \mu \mathrm{m}$ FSW. After $24 \mathrm{~h}, 3 \times 10^{6}$ untreated Symbiodinium cells (positive control, POS) or similar numbers of Symbiodinium from one of five cell surface modification treatments were separately added to three replicate containers per treatment, resulting in a concentration of $1.5 \times 10^{4}$ cells $/ \mathrm{mL}$. A negative control was also employed, i.e., larvae in containers without Symbiodinium cells. Fifteen coral larvae were sampled randomly from each of the three containers per treatments at 12, 24, 48, 72 and $96 \mathrm{~h}$ post

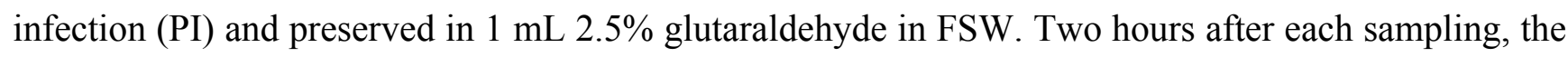
fixative was removed and the coral larvae were washed 3 times then stored in $1 \mathrm{~mL} 0.1 \mathrm{M}$ phosphate buffer $\left(0.2 \mathrm{M} \mathrm{NaH}_{2} \mathrm{PO}_{4}, 0.2 \mathrm{M} \mathrm{Na}_{2} \mathrm{HPO}_{4}\right.$ and $\left.\mathrm{pH}=7.2\right)$. The proportion of larvae infected with Symbiodinium and the cell abundance within larvae were quantified by counting the number of Symbiodinium cells in 15 larvae per tub per treatment (total $n$ per treatment $=45$ larvae). Larvae were squashed under a cover slip and symbiont cells were counted at $20 \times$ magnification under fluorescent light (Axioskop 2 Zeiss). Samples of 20 larvae from all surface treatments were preserved in 100\% $\mathrm{EtOH}$ at the end of the experiment. These samples were analysed using SSCP of ITS- 1 to confirm that only a single Symbiodinium type (C1 or D) had been taken up [52].

\subsection{Statistical Analysis}

The number of larvae infected and the level of infection (density of Symbiodinium cells per larva) were analysed using bootstrap-based ANOVA [55]. The assumption of homoscedastic variances required for ANOVA was violated and transformations did not rectify this problem. The observed $F$-statistics were therefore tested against the distribution of $F$-statistics resulting from 4999 randomisations of the data. The negative control treatment never resulted in infection, and was therefore not included in the analyses. To test whether symbiont abundance per larva were independent at the experimental 
container level, we first conducted a random bootstrap-based one-way ANOVA. Because infection levels did not vary significantly among larvae within an experimental container $(\mathrm{F}=1.69 ; p=0.19)$, this level was omitted from further analyses. However, the number of infected larvae did vary among experimental containers within a treatment $(\mathrm{F}=0.71 ; p=0.007)$, and therefore the experimental container was used as the unit of replication.

Three-way random bootstrap-based ANOVAs were used to test whether the abundance of Symbiodinium cells per larva and the number of larvae infected per sample differed between Symbiodinium types $(n=2)$, or among surface treatments $(n=6)$ or sample times $(n=5)$. This analysis is appropriate for proportional data when test distributions are generated via bootstrapping $[55,56]$. The model used contained all interaction terms among the following main effects: $S=\mu+\alpha+\beta+\gamma+\varepsilon$, where $S$ is the number of Symbiodinium cells per larva or the number of larvae infected per sample ( $\alpha, \beta$ and $\gamma$ are the main effects of Symbiodinium type, time and treatment, and $\varepsilon$ the error). Post-hoc comparisons were conducted using Matlab's multcompare function [57]. This function performs multiple comparisons while correcting for repeated tests. Tukey's HSD criterion was used to provide the correction [58,59]. To reveal within-type differences potentially obscured by large overall differences between Symbiodinium C1 and D, post-hoc analyses were conducted separately for each symbiont type. Differences in the proportion of dead cells between C1 and D type Symbiodinium extractions were tested using a two-way bootstrap-based ANOVA (Time = 0, 24, 48, 72 h; Type = C1, D) and post-hoc analyses as described above.

\section{Results}

\subsection{Differences in Infection Dynamics between C1 and D Symbiodinium}

The mean abundance of Symbiodinium cells taken up by coral larvae in the first $96 \mathrm{~h}$ following symbiont addition and the mean proportion of larvae infected per treatment differed significantly between Symbiodinium types (Table 1 and 2). The proportion of larvae infected by C1 was almost $100 \%$, whereas only $29-71 \%$ of larvae exposed to D type Symbiodinium were infected at the end of the experiment. Post-hoc comparisons revealed a more than five-fold greater mean $( \pm \mathrm{SE})$ abundance of symbionts in larvae offered C1 $(45.64 \pm 2.36)$ than in those offered D Symbiodinium $(1.05 \pm 0.17)$. The process of freshly extracting cells from coral hosts affected Symbiodinium types differently, resulting in significantly higher, albeit still relatively low, mean $( \pm \mathrm{SE})$ proportions of dead cells for $D$ $(5.8 \pm 2.3 \%$ dead cells) compared to C1 Symbiodinium $(1 \pm 0.41 \%$ dead cells) (Figure 1; Table 3$)$. 
Table 1. Three-factor orthogonal ANOVA comparing the mean abundance of Symbiodinium cells in larvae of Acropora tenuis between symbiont types (C1 and D), among cell surface treatments (two enzymes, three lectins and unmodified controls) and sampling times $(12,24,48,72$ and $96 \mathrm{~h})$.

\begin{tabular}{lccccc}
\hline Source & SS & d.f. & Mean Sq & Observed F & Prob $>$ F \\
\hline Symbiodinium type & 1827020 & 1 & 1827020 & 583.1 & $<0.001$ \\
Treatment & 355979 & 5 & 71196 & 22.7 & $<0.001$ \\
Time & 1638229 & 4 & 409557 & 130.7 & $<0.001$ \\
Type*Treatment & 384978 & 5 & 76996 & 24.6 & $<0.001$ \\
Type*Time & 1524336 & 4 & 381084 & 121.6 & $<0.001$ \\
Treatment*Time & 551839 & 20 & 27592 & 8.8 & $<0.001$ \\
Type*Treatment*Time & 579771 & 20 & 28989 & 9.3 & $<0.001$ \\
\hline Error & 8272171 & 2640 & 3133 & & \\
\hline
\end{tabular}

$\mathrm{SS}=$ Sums of Squares, Mean $\mathrm{Sq}=$ Mean squares, Prob $>\mathrm{F}=$ probability of $\mathrm{F}$ being greater than the distribution of F-values obtained through randomisation.

Table 2. Three-factor orthogonal ANOVA comparing the proportion of $A$. tenuis larvae infected with Symbiodinium cells between symbiont types (C1 and D), among cell surface treatments (two enzymes, three lectins and unmodified controls) and sampling times $(12,24,48,72$ and $96 \mathrm{~h})$.

\begin{tabular}{lccccc}
\hline Source & SS & d.f. & Mean Sq. & Observed F & Prob $>$ F \\
\hline Symbiodinium type & 7.52 & 1 & 7.524 & 174.5 & $<0.001$ \\
Treatment & 0.25 & 5 & 0.0504 & 1.2 & 0.330 \\
Time & 10.09 & 4 & 2.524 & 58.5 & $<0.001$ \\
Type*Treatment & 0.10 & 5 & 0.0204 & 0.5 & 0.800 \\
Type*Time & 0.70 & 4 & 0.175 & 4.1 & 0.003 \\
Treatment*Time & 0.89 & 20 & 0.045 & 1.0 & 0.444 \\
Type*Treatment*Time & 1.57 & 20 & 0.078 & 1.8 & 0.004 \\
\hline Error & 5.18 & 120 & 0.043 & & \\
\hline
\end{tabular}

$\mathrm{SS}=$ Sums of Squares, Mean $\mathrm{Sq}=$ Mean squares, Prob $>\mathrm{F}=$ probability of $\mathrm{F}$ being greater than the distribution of F-values obtained through randomisation.

Table 3. Two-factor orthogonal ANOVA comparing the proportion of dead $\mathrm{C} 1$ and $\mathrm{D}$ Symbiodinium cells among sampling times $(0,24,48$ and $72 \mathrm{~h})$.

\begin{tabular}{lccccc}
\hline Source & SS & d.f. & Mean Sq. & Observed F & Prob $>$ F \\
\hline Symbiodinium type & 36.86 & 1 & 36.86 & 8.61 & 0.007 \\
Time & 10.09 & 3 & 10.47 & 2.45 & 0.089 \\
Type*Time & 26.80 & 3 & 8.69 & 2.03 & 0.136 \\
\hline Error & 102.71 & 24 & 4.27 & & \\
\hline
\end{tabular}

$\mathrm{SS}=$ Sums of Squares, Mean $\mathrm{Sq}=$ Mean squares, Prob $>\mathrm{F}=$ probability of $\mathrm{F}$ being greater than the distribution of F-values obtained through randomisation. 
Figure 1. Comparison of mean percentage of dead cells between $\mathrm{C} 1$ and D Symbiodinium at four times after extraction. Grey bars = type $\mathrm{C} 1($ mean $\pm \mathrm{SEM})$; white bars = type D (mean \pm SEM).

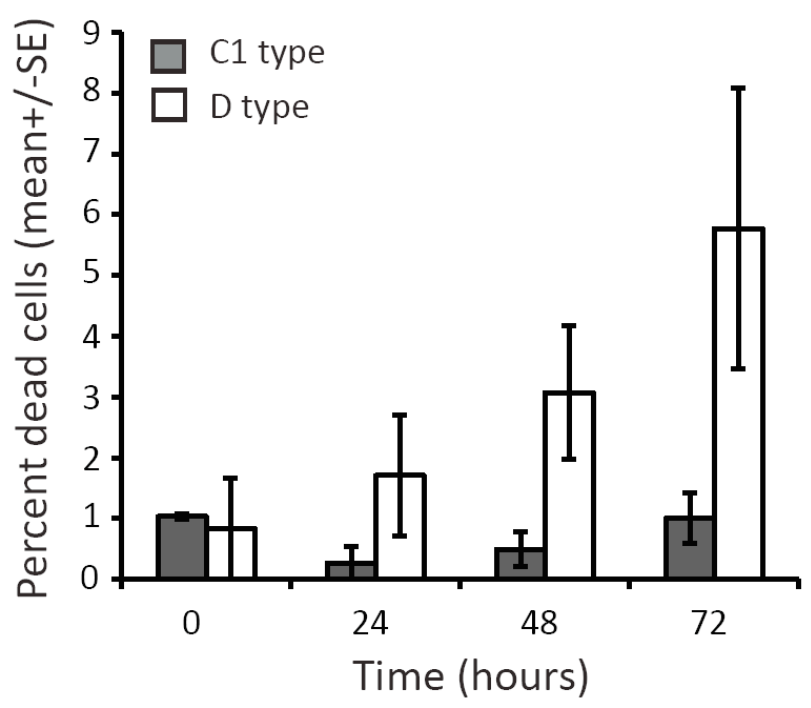

\subsection{Cell Surface Recognition and the Onset of Symbiosis}

Treatments that modified Symbiodinium cell surface molecules significantly affected the number of cells in larvae (Table 1) but not the proportions of larvae infected (Table 2). Variation in C1 symbiont abundance within larvae was high among treatments, and notably, significant differences were found between enzyme-treated versus both untreated and lectin-treated cell surfaces for this symbiont type (Figure 2a). In general, larvae contained significantly more algal cells when they were treated with the enzymes $\alpha$-Amylase [AA] and Trypsin, which modified glyco-protein surface exudates, compared to unmodified Symbiodinium cells (Figure 2a). In our larval study, the numbers of Symbiodinium C1 cells taken up when cells were treated with the lectins ConA and WGA were similar to those of unmodified cells, but significantly lower than numbers of enzyme-treated cells (Figure 2a). In contrast to patterns of infection found for Symbiodinium C1, larvae supplied with Symbiodinium D had greater mean levels of infection at $96 \mathrm{~h}$ when supplied with untreated and lectin-treated cells compared to enzyme-treated cells, although post hoc tests did not indicate that differences were statistically significant overall (Figure 2b).

Confocal microscopy revealed that the ConA and WGA lectins used here bind to the cell surface of both Symbiodinium cell types, either in localised areas or as a smooth layer surrounding Symbiodinium cells (Figure 3b, d, g, i). The absence of corresponding fluorescent patterns in control cells (Figure 3a, f) and reductions in localised areas of fluorescence when inhibitors were added to lectin treatments (Figure 3c, e, h, j) confirm that the fluorescent patterns observed are attributable to the lectins. These lectin binding patterns are consistent with those detected by Logan et al. [29] on cultured material and support lectin specificity for algal fractions rather than host debris in fresh isolates. Overall, lectin labelling was high (76-85\%) in three treatments, but was only 25\% for C1-type cells labelled with ConA (Figure 3b). In comparison, 10-32\% of cells were labelled with lectins pre-exposed to an inhibitor (Figure 3c, e, h, j), suggesting incomplete inhibition and/or non-specific labelling at levels similar to those detected by flow cytometry by Wood-Charlson et al. [30]. 
Figure 2. Mean number of (a) C1 and (b) D Symbiodinium cells per larva $(\mathrm{n}=45)$. Statistical significance of pairwise post-hoc comparisons among sampling times and cell surface treatments are presented in boxes ( + indicates significance at $\alpha=0.05$ ). AA $=\alpha$-Amylase, TRY $=$ Trypsin, POS $=$ Positive unmodified control, ConA $=$ Concanavilin A lectin from Jack bean, LPA = lectin from Limulus polyphemus, WGA = lectin from Triticum vulgaris.

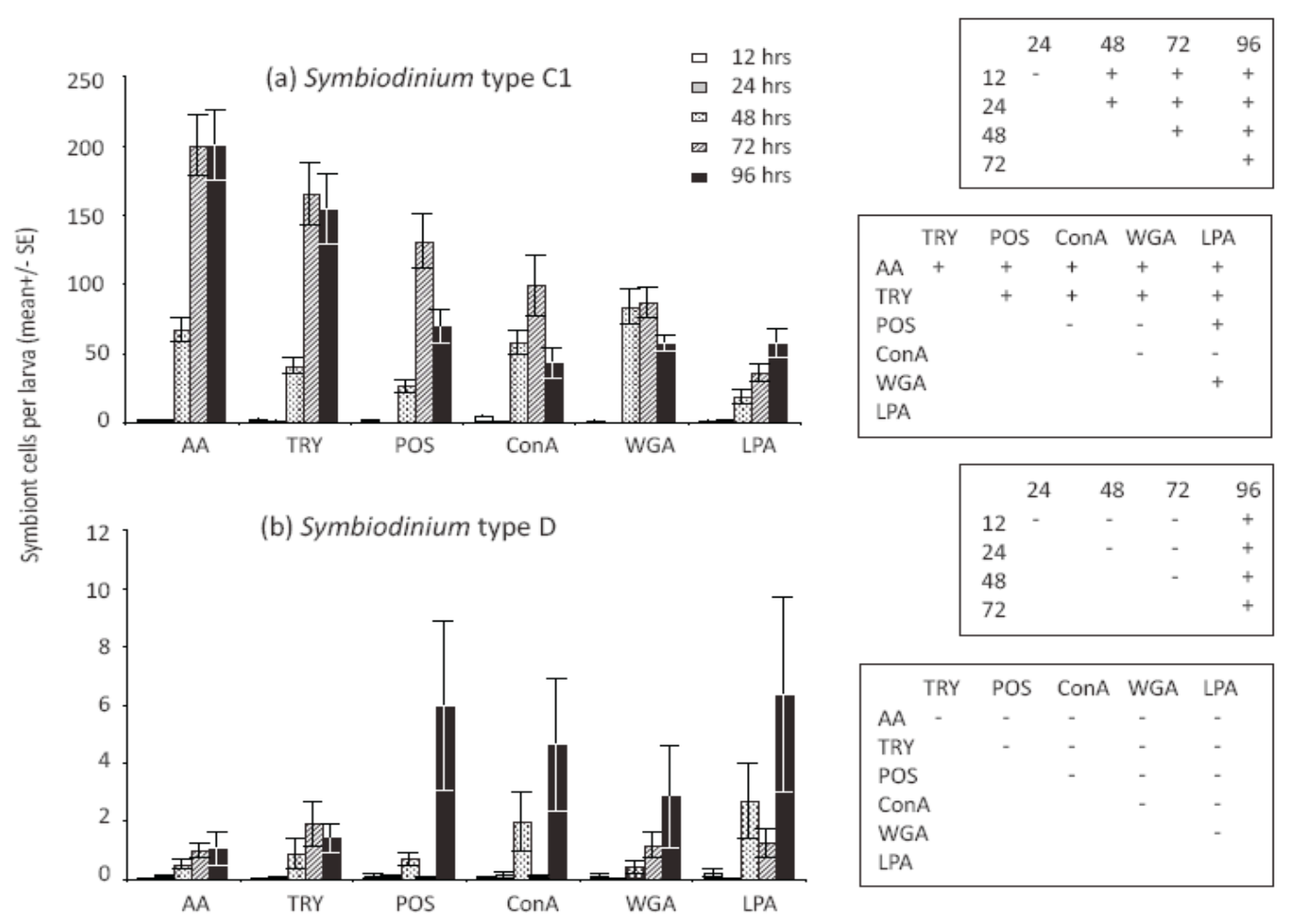

Figure 3. Representative confocal images of lectin-labelled (as indicated by green fluorescence) and unmodified (control) Symbiodinium cells for the following treatments to C1/D cells: (a/f) unmodified control cells; (b/g) ConA treated cells; (c/h) ConA plus inhibitor treated cells; (d/i) WGA treated cells; and (e/j) WGA + inhibitor treated cells. Percent of cells labelled indicated in bottom right corner All scale bars $=10 \mu \mathrm{m}$. 1 = Accumulation body, 2 = articulated chloroplast, 3 = Bound Alexa488 fluorolabelled lectin. ConA = Concanavilin A lectin, WGA = lectin from Triticum vulgaris.
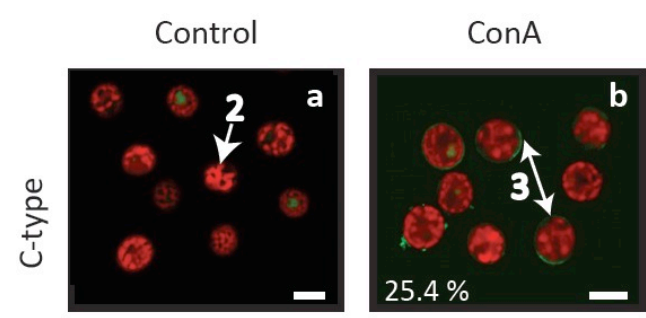

ConA + Inhib

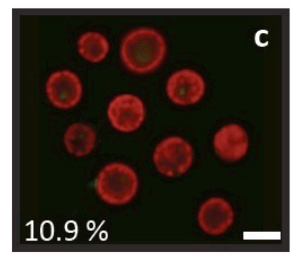

WGA

WGA + Inhib
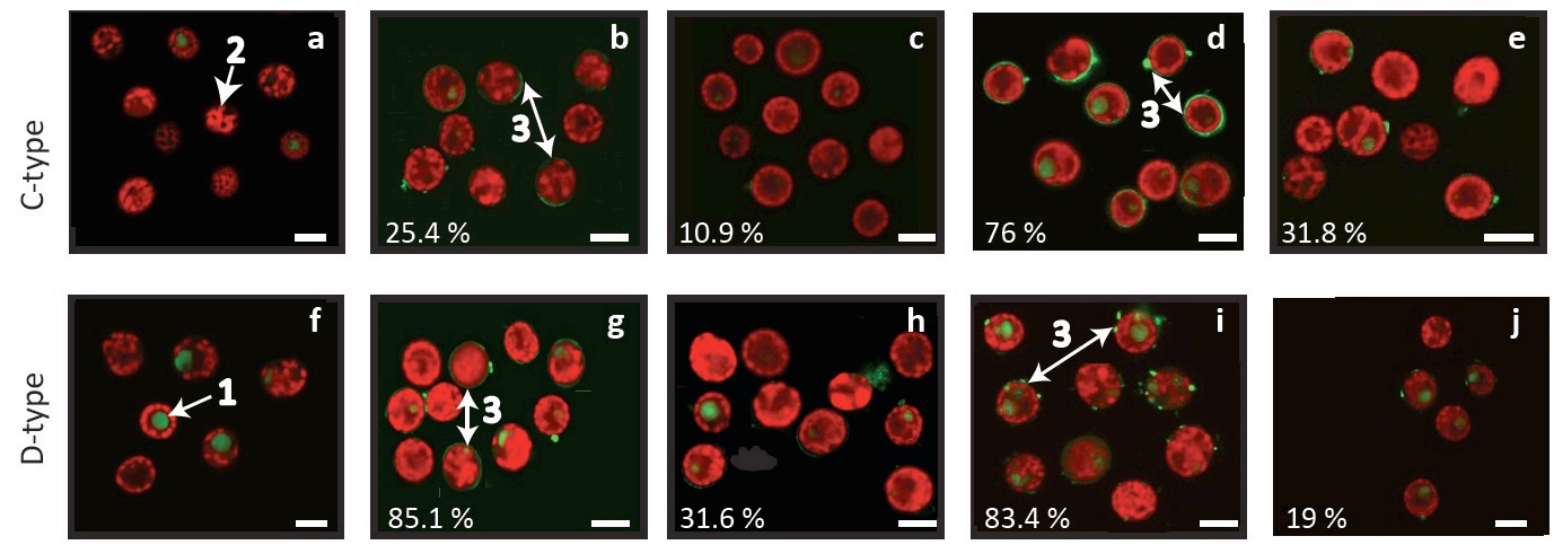


\subsection{Temporal Dynamics of Symbiodinium Infection in Acropora Tenuis Larvae}

We detected significant changes in densities of both Symbiodinium types and the proportion of larvae infected in the first $96 \mathrm{~h}$ (Table 1 and 2). The proportion of larvae infected with $\mathrm{C} 1$ Symbiodinium differed significantly among earlier but not later sampling times for type-C1, and almost all larvae were infected in all treatments after $48 \mathrm{~h}$ (Figure $4 \mathrm{a}$ ). The proportions of larvae infected with Symbiodinium D were lower and more variable across all treatments, and post-hoc comparisons revealed a significant increase in the proportions of D infected larvae at $48 \mathrm{~h}$ compared to earlier and later time points (Figure 4b). While almost $100 \%$ of larvae were infected with Symbiodinium C1 at $48 \mathrm{~h}$ (Figure 4a), post hoc analyses of cell densities revealed significant overall increases at $48 \mathrm{~h}$ and further changes at 72 and $96 \mathrm{~h}$ (Figure 2a). In contrast, overall cell densities in larvae exposed to Symbiodinium D did not increase significantly until 96 h (Figure 2b). Interestingly, Symbiodinium C1 cell densities declined for larvae exposed to untreated Symbiodinium (POS: $p=0.009$ ) and two lectin-blocked cell extracts (ConA: $p=0.02$ and WGA: $p=0.03$ ) between 72 and $96 \mathrm{~h}$ but not in the remaining cell surface treatments (AA: $p=0.92$; LPA: $p=0.08$; Trypsin: $p=0.74$ ). Conversely, Symbiodinium D cells increased in abundance within larvae between 72 and $96 \mathrm{~h}$ in ConA treated and unmodified cells (ConA: $\mathrm{p}<0.001$; POS: $\mathrm{p}=0.02)$ but not in the remaining treatments (AA: $\mathrm{p}=0.96$; LPA: $p=0.13$; WGA: $p=0.44$; Trypsin: $p=0.66$ ).

Figure 4. Proportions of infected larvae using (a) C1 and (b) D Symbiodinium cells ( $\mathrm{n}=3$ ). Statistical significance of pairwise post-hoc comparisons among sampling times and cell surface treatments are presented in boxes ( + indicates significance at $\alpha=0.05$ ). $\mathrm{AA}=\alpha$-Amylase, $\mathrm{TRY}=$ Trypsin, $\mathrm{POS}=$ Positive unmodified control, ConA $=$ Concanavilin A lectin from Jack bean, LPA = lectin from Limulus polyphemus, WGA = lectin from Triticum vulgaris.

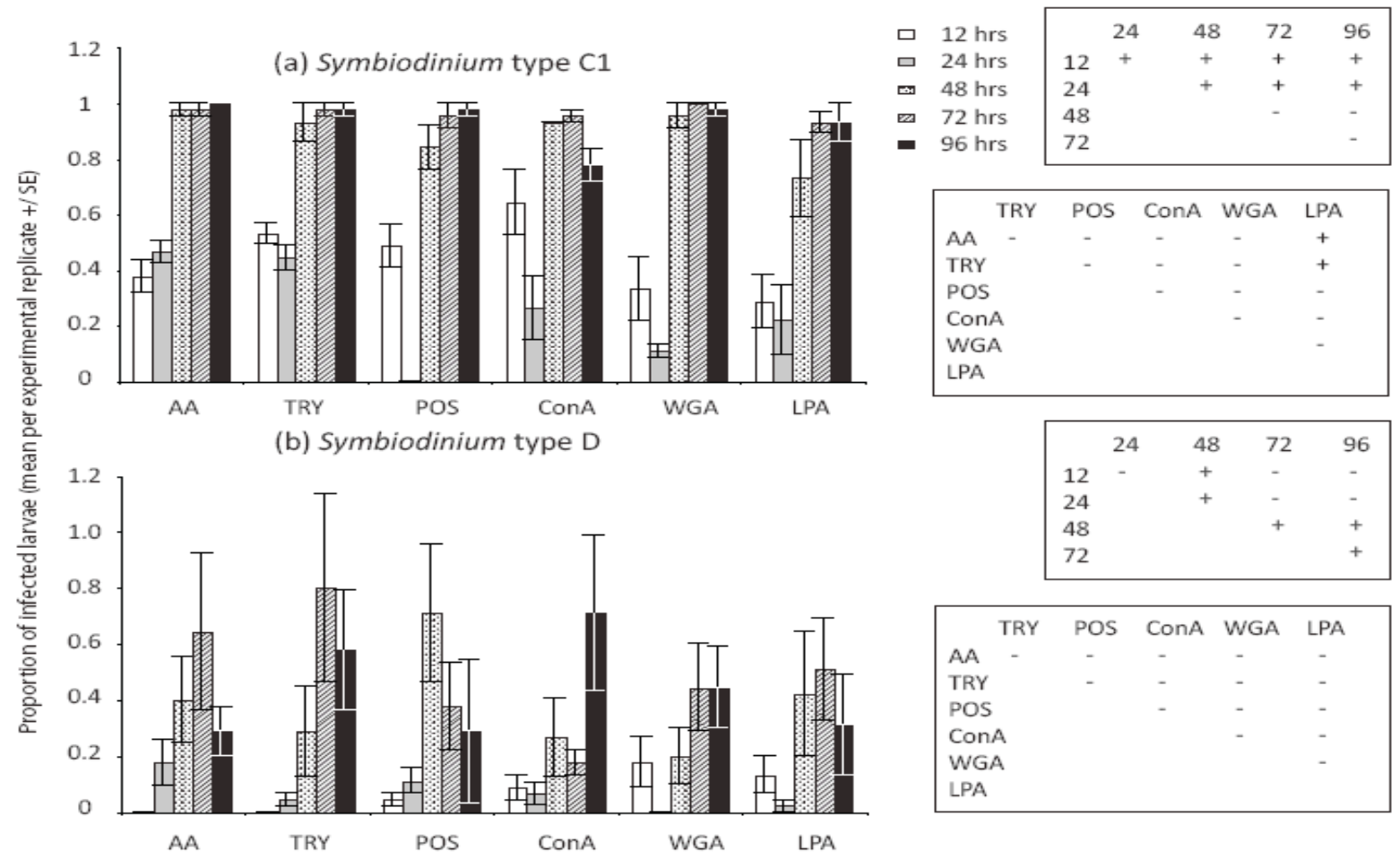




\section{Discussion}

\subsection{Differences in Infection Dynamics Between $C 1$ and D Symbiodinium}

This study found significant differences in the degree to which A. tenuis larvae procured $\mathrm{C} 1$ compared to D Symbiodinium in the early establishment stages of symbiosis, leading to a higher proportion of larvae infected and up to a five-fold greater abundance of C1 Symbiodinium compared to D Symbiodinium after four days of symbiont exposure. In contrast to our finding of greater initial procurement of C1 Symbiodinium by larvae, one-month-old A. tenuis juveniles, naturally inoculated and grown at the parental location (Magnetic Island), host mostly Symbiodinium D for up to 2 years [7,47]. Differences in symbiont patterns between larvae and juveniles could arise from differences in infectivity of symbiont types and/or post-infection growth rate, both potentially affected by host "winnowing" mechanisms and local environmental conditions [2,12,23,24,46,60]. The greater larval uptake of the Symbiodinium type dominating adult populations of A. tenuis (i.e., Symbiodinium C1) found here accords with results found for other coral species. In Fungia scutaria, for example, the most robust symbiosis occurs when larvae were exposed to the Symbiodinium type dominating adult F. scutaria populations (i.e., C1f) [46,60,61]. Conversely, differences in infection levels may also exist because of symbiont dynamics. For example, Symbiodinium A from Montastraea annularis and Tridacnea crocera has been described as "weedy" because of its higher high stress tolerance, superior ability to infect larvae, and greater post-infection growth rates compared to the more variable C type $[10,43,62]$. Prevailing environmental conditions also affect rates of uptake and proliferation of different Symbiodinium types [12]. Symbiodinium C1 is more infective and proliferates more readily within Acropora larvae at temperatures between $25-28{ }^{\circ} \mathrm{C}$, with infection rates decreasing significantly at temperatures above $29^{\circ} \mathrm{C}$ [63]. In contrast, uptake of Symbiodinium D is unaffected by temperature, with low initial uptake increasing over time at temperatures between $26-32{ }^{\circ} \mathrm{C}$ [63]. Our study was conducted at $27{ }^{\circ} \mathrm{C}$, which promotes uptake and proliferation of Symbiodinium $\mathrm{C} 1$, potentially explaining the higher proportion of larvae infected by Symbiodinium C1 and its high abundances within $A$. tenuis larvae.

An alternative explanation for differences in initial infection and/or post-infection growth found between Symbiodinium cells could arise as a consequence of processes used to freshly extract Symbiodinium cells from donor coral tissues. A follow-up test found that extraction processes compromised Symbiodinium D significantly more than Symbiodinium C1 cells, although mortality of Symbiodinium D was only $6 \%$ after 72 h (i.e., a loss of 900 cells). Although we cannot discount the possibility that differences in infection dynamics between $\mathrm{D}$ and $\mathrm{C} 1$ symbionts were a consequence of compromised health of D cells, the magnitude of the differences found, both in the proportion of larvae infected and in cell densities between the two Symbiodinium types, suggest that it is unlikely that they were due to Symbiodinium health alone. The difficulty in culturing many Symbiodinium types justifies the use of freshly extracted Symbiodinium in experimental research on corals, however extraction effects must be (but rarely are) quantified because of their potential to affect infection dynamics. For example, Weis et al. [46] found that repeated washing of freshly isolated Symbiodinium cells from four coral sources reduced their ability to establish symbiosis with Fungia scutaria. To correctly assign differences in infection and post-infection growth among Symbiodinium types, cultures of ecologically 
relevant, similarly acclimated types must be used. If fresh extraction cannot be avoided, their health and viability must be accounted for using techniques such as PAM fluorometry and vital stains.

\subsection{Cell Surface Recognition and the Onset of Symbiosis within C1 and D Symbiodinium}

Treatments that modified Symbiodinium cell surface molecules significantly affected the number of cells in larvae, but not the proportion of larvae infected. This suggests that Symbiodinium cell surface molecules are involved in post-phagocytosis, rather than pre-phagocytosis recognition $[12,36,42,63]$. Coral larvae are promiscuous and initially associate with multiple types of Symbiodinium, suggesting that their pre-phagocytosis recognition system is non-specific. Little is known about post-phagocytosis processes, but the high abundance of symbionts found in larvae exposed to enzymatically treated $\mathrm{C} 1$ Symbiodinium cells suggests that the 40-100 kD glycome, including glycoproteins and long chain starch residues, plays a role in controlling post-phagocytic survival and growth of $\mathrm{C} 1$ symbionts within coral larvae. Our findings contrast with previous studies, which have uniformly found lower (or similar) infection levels with enzyme-treated compared to untreated Symbiodinium cells [30,31]. Differences in ontogenetic patterns of symbiosis among corals may explain these contrasting results. Coral species examined in previous studies were dominated by the same symbiont type in both the juvenile and adult life stages [30,31], whereas two symbiont types occur in A. tenuis and vary in dominance between juvenile and adult corals. We hypothesise that cell surface recognition molecules may differ among Symbiodinium types and vary in their capacity to maintain symbiosis in different life stages of the coral. However, the hypothesis that surface recognition molecules can limit uptake or post-infection cell proliferation in flexible coral-Symbiodinium symbioses requires further testing in a range of species and environmental conditions.

Interestingly, the LPA lectin treatment resulted in significantly lower numbers of Symbiodinium C1 cells compared to all other treatments but the ConA treatment, indicating that $N$-acetyl neuraminic acid, glycuronic acid and/or phosphorylcholine sugar moieties on Symbiodinium C1 cell surfaces are involved in early recognition of compatible Symbiodinium cells by the coral host. Conversely, the similarity in infection levels among WGA-treated, ConA-treated and untreated cells suggests that $\alpha$-mannose, $\alpha$-glycose, $N$-acetyl- $\beta$-D-glycosaminyl and/or $N$-acetyl- $\beta$-D-glycosamine sugar residues on Symbiodinium cell surfaces do not play an important role in determining levels of early uptake under our laboratory conditions. This result is surprising, because these sugars commonly occur on the surfaces of a range of micro-algae [28-30,34], and have been implicated in cellular recognition [28-32,34]. The presence of a range of sugar molecules can vary among cell stages, species and geographical locations [28,64], potentially explaining the results obtained here. We exposed coral larvae to Symbiodinium cells for the duration of the experiment, during which time cell surface molecules may have changed. For example, Aguilera and Gonzáles-Gil [28] found that while $\alpha$-mannose, $\alpha$-glycose and $\mathrm{N}$-acetyl residues were common and relatively stable on the surface of four dinoflagellate species (but not Symbiodinium spp), they were reduced or absent at certain sampling times during the 52-hour experimental period. In contrast, Logan et al. [29] found that all eight Symbiodinium cultures tested displayed significant binding to ConA at all sampling times (3.5 and 5.5 weeks after sub-culturing, repeated over four culture cycles) indicating the widespread and stable presence of mannose residues on these cell types, assuming specificity of the ConA probe. Likewise, $50 \%$ of cell types tested bound 
equally well to WGA at all sampling times, demonstrating the common presence of $N$-acetyl residues on the cell surfaces of cultured Symbiodinium types. Our results confirm the presence of both mannose and $\mathrm{N}$-acetyl groups on both the $\mathrm{C} 1$ and D-type Symbiodinium used here. If these molecules are temporally stable in the $\mathrm{C} 1$ and D Symbiodinium types studied here, then our results suggest that these residues are less important in the early onset of this symbiosis compared to long chain starch and glyco-proteins. It is also possible that other glycans, not tested here, may be important for cell recognition in A. tenuis. For example, lectins specific for galactose-residues (termed galectins) bound to most Symbiodinium types tested by Logan et al. [29] and may be important in the maintenance of successful symbioses by keeping Symbiodinium cells in a non-motile phase [65]. Lin et al. [31] and Wood-Charlson et al. [30] detected a reduced uptake of Symbiodinium cells whose galactose residues had been obscured. Detailed characterisations of the glycomes of Symbiodinium populations and types, for both freshly extracted and cultured Symbiodinium material (as demonstrated by [29]), combined with examinations of the ability of respective Symbiodinium cells to establish symbiosis with coral species will be required to further explore the role of cellular recognition in onset and maintenance of coral-Symbiodinium symbiosis.

\subsection{Temporal Dynamics of Symbiodinium Infection in Acropora tenuis Larvae}

The temporal scale over which infection by Symbiodinium cells occurs varies among coral species. In Fungia scutaria, larvae are infected within a few h, after which densities of cells remain relatively constant $[46,60]$. In contrast, we detected significant variation in the temporal infection dynamics of C1 and D type Symbiodinium used here. While almost $100 \%$ of larvae were infected with Symbiodinium $\mathrm{C} 1$ after two days overall cell densities in larvae exposed to Symbiodinium D did not increase significantly until four days after symbiont exposure. Interestingly, $\mathrm{C} 1$ cell densities declined between days three and four in larvae exposed to untreated Symbiodinium and to two out of three lectin-blocked cell extracts. In contrast, Symbiodinium D cells increased in abundance over the same time scale within larvae exposed to ConA treated and unmodified cells, possibly suggesting the initiation of a winnowing process involving specific glycan-lectin interactions. The time frame over which these declines in $\mathrm{C} 1$ and increases in D occurred is similar to delays in cell "sorting" processes reported for Hydra endosymbiosis, cell sorting being the process by which Hydra modifies their Chlorella complement through disintegration or ejection of cells [66]. Similarly, Davy et al. [23] found that both homologous and heterologous symbiont densities declined four days after uptake, but ultimately increased and stable symbioses were formed with both types 36 weeks after infection. Our results suggest that glycan-lectin interactions, in particular those involving $N$-acetyl residues, may be associated with the initiation of a winnowing mechanism after only four days and, if continued, may explain the dominance of the $\mathrm{D}$ type in juveniles of $A$. tenuis [7,36]. To elucidate the complex recognition mechanisms that corals may use to shape their symbiont communities during the establishment of symbiosis and subsequent winnowing process, future studies should examine infection dynamics over an extended temporal scale. 


\section{Conclusions}

In summary, our results suggest that glycoprotein exudates and molecules on the surface of Symbiodinium cells play a role in controlling post-infection growth of Symbiodinium C1. Reduced proliferation of Symbiodinium C1 when some lectin receptor sites were blocked suggests that specific sugar moieties ( $\mathrm{N}$-acetyl neuraminic acid, glycuronic acid and/or phosphorylcholine) are involved in recognition of this symbiont type in hospite. Higher abundance of symbiont cells with modified glycoproteins and long chain starch residues compared to the control treatment suggests that the absence of these cues promotes the proliferation of Symbiodinium $\mathrm{C} 1$ cells. The combination of several techniques, including transcriptomic analysis of symbiosis [67,68], high throughput glycome characterisation [27], as well as increased ability to culture local Symbiodinium types, will enable an increased understanding of the molecular and cellular mechanisms underlying establishment of coral-Symbiodinium symbioses in the future.

\section{Acknowledgments}

This study was conducted under GBRMPA permit G06_20473.1. Funding was provided by ARC Centre of Excellence and the Queensland Government using facilities at Australian Institute of Marine Science and James Cook University. We thank Reanna Willis and Hannah Jensen for assistance in data collection, V Beltran-Ramirez for quantifying cell mortality, AH Baird and five anonymous reviewers for their insightful comments on earlier versions of this manuscript.

\section{References}

1. Douglas, A.E. Host benefits and the evolution of specialization in symbiosis. Heredity 1998, 81, 599-603.

2. Nyholm, S.V.; McFall-Ngai, M.J. The winnowing: Establishing the Squid-Vibrio symbiosis. Nat. Rev. Microbiol. 2004, 2, 632-642.

3. Smith, S.E.; Read, D.J. Mycorrhrizal Symbiosis; Academic Press: London, UK, 1997; pp. 1-653.

4. Muscatine, L. The role of Endosymbiotic Algae in Carbon and Energy Flux in Reef Corals. In Ecosystems of the World: Coral Reefs; Dubinsky, Z., Ed.; Elsevier: Amsterdam, The Netherlands, 1990; pp. 75-88.

5. Marquez, L.M.; Redman, R.S.; Rodriguez, R.J.; Roossinck, M.J. A virus in a fungus in a plant: Three-way symbiosis required for thermal tolerance. Science 2007, 315, 513-515.

6. Cantin, N.E.; van Oppen, M.J.H.; Willis, B.L.; Mieog, J.C.; Negri, A.P. Juvenile corals can acquire more carbon from high performance algal endosymbionts. Coral Reefs 2009, 28, 405-414.

7. Little, A.F.; van Oppen, M.J.H.; Willis, B.L. Flexibility in algal endosymbioses shapes growth in reef corals. Science 2004, 304, 1492-1494.

8. Berkelmans, R.; van Oppen, M.J.H. The role of zooxanthellae in the thermal tolerance of corals: A 'nugget of hope' for coral reefs in an era of climate change. Proc.Biol. Sci. 2006, 273, 2305-2312.

9. Abrego, D.; Ulstrup, K.E.; Willis, B.L.; van Oppen, M.J.H. Species-specific interactions between algal endosymbionts and coral hosts define their bleaching response to heat and light stress. Proc. Biol. Sci. 2008, 275, 2273-2282. 
10. Rowan, R.; Knowlton, N.; Baker, A.; Jara, J. Landscape ecology of algal symbionts creates variation in episodes of coral breaching. Nature 1997, 388, 265-269.

11. Hughes, T.P.; Baird, A.H.; Bellwood, D.R.; Card, M.; Connolly, S.R.; Folke, C.; Grosberg, R.; Hoegh-Guldberg, O.; Jackson, J.B.C.; Kleypas, J. Climate change, human impacts, and the resilience of coral reefs. Science 2003, 301, 929-933.

12. Baird, A.H.; Cumbo, V.R.; Leggat, W.; Rodriguez-Lanetty, M. Fidelity and flexibility in coral symbioses. Mar. Ecol. Prog. Ser. 2007, 347, 307-309.

13. Harrison, P.L.; Wallace, C.C. Reproduction, dispersal and recruitment of scleractinian corals. In Ecosystems of the World, Coral Reefs; Dubinsky, Z., Ed.; Elsevier: Amsterdam, The Netherlands, 1990; Volume 25, pp. 133-207.

14. Baird, A.H.; Guest, J.R.; Willis, B.L. Systematic and biogeographical patterns in the reproductive biology of scleractinian corals. Annu. Rev. Ecol. Evol. Syst. 2009, 40, 551-571.

15. Mieog, J.C.; van Oppen, M.J.H.; Cantin, N.E.; Stam, W.T.; Olsen, J.L. Real-time PCR reveals a high incidence of Symbiodinium clade D at low levels in four scleractinian corals across the Great Barrier Reef: Implications for symbiont shuffling. Coral Reefs 2007, 26, 449-457.

16. Jones, A.M.; Berkelmans, R.; van Oppen, M.J.H.; Mieog, J.C.; Sinclair, W. A community change in the algal endosymbionts of a scleractinian coral following a natural bleaching event: Field evidence of acclimatization. Proc.Biol. Sci. 2008, 275, 1359-1365.

17. Jolley, E.; Smith, D.C. The green hydra symbiosis. II. The biology of the establishment of the association. Proc.Biol. Sci. 1980, 207, 331-333.

18. Muscatine, L.; Pool, R.R.; Trench, R.K. Symbiosis of algae and invertebrates: Aspects of the symbiont surface and the host-symbiont interface. Trans. Am. Microsc. Soc. 1975, 94, 450-469.

19. Markell, D.A.; Trench, R.K. Macromolecules exuded by symbiotic dinoflagellates in culture: Amino acid and sugar composition. J. Phycol. 1993, 29, 64-68.

20. Markell, D.A.; Wood-Charlson, E.M. Immunocytochemical evidence that symbiotic algae secrete potential recognition signal molecules in hospite. Mar. Biol. 2010, 157, 1105-1111.

21. Colley, N.J.; Trench, R.K. Selectivity in phagocytosis and persistence of symbiotic algae by the scyphistoma stage of the jellyfish Cassiopeia xamachana. Proc.Biol. Sci. 1983, 219, 61-82.

22. Colley, N.J.; Trench, R.K. Cellular events in the reestablishment of a symbiosis between a marine dinoflagellate and a coelenterate. Cell Tissue Res. 1985, 239, 93-103.

23. Davy, S.K.; Lucas, I.A.N.; Turner, J.R. Uptake and persistence of homologous and Heterologous zooxanthellae in the temperate sea anemone Cereus pedunculatus (Pennant). Biol. Bull. 1997, 192, 208-216.

24. Schoenberg, D.A.; Trench, R.K. Genetic variation in Symbiodinium (= Gymnodinium) microadriaticum Freudenthal, and specificity in its symbiosis with marine invertebrates. III. Specificity and infectivity of Symbiodinium microadriaticum. Proc.Biol. Sci. 1980, 207, 445-460.

25. Coffroth, M.A.; Santos, S.R.; Goulet, T.L. Early ontogenetic expression of specificity in a cnidarian-algal symbiosis. Mar. Ecol. Prog. Ser. 2001, 222, 85-96.

26. Weis, V.M. Cellular mechanisms of Cnidarian bleaching: Stress cause a breakdown of symbiosis. J. Exp. Biol. 2008, 211, 3059-3066.

27. Laughlin, S.T.; Bertozzi, C.R. Imaging the glycome. Proc. Natl. Acad. Sci. USA 2009, 106, 12-17. 
28. Aguilera, A.; Gonzáles-Gil, S. Lectin analysis of surface saccharides during the cell cycle in four dinoflagellate species. J. Exp. Mar. Biol. Ecol. 2001, 256, 149-166.

29. Logan, D.D.K.; LaFlamme, A.C.; Weis, V.M.; Davy, S.K. Flow-cytometric characterisation of the cell-surface glycans of symbiotic dinoflagellates (Symbiodinium spp.). J. Phycol. 2010, 46, 525-533.

30. Wood-Charlson, E.M.; Hollingsworth, L.L.; Krupp, D.A.; Weis, V.M. Lectin/glycan interactions play a role in recognition in a coral/dinoflagellate symbiosis. Cell Microbiol. 2006, 8, 1985-1993.

31. Lin, K.L.; Wang, J.T.; Fang, L.S. Participation of glycoproteins on zooxanthellal cell walls in the establishment of a symbiotic relationship with the sea anemone, Aiptasia pulchella. Zool. Stud. 2000, 39, 172-178.

32. Kvennefors, C.E.; Leggat, W.; Hoegh-Guldberg, O.; Degnan, B.M.; Barnes, A.C. An ancient and variable mannose-binding lectin from the coral Acropora millepora binds both pathogens and symbionts Dev. Comp. Immunol. 2008, 32, 1582-1592.

33. Goldstein, I.J.; Hughes, R.C.; Monsigny, M.; Osawaand, T.; Sharon, N. What should be called a lectin? Nature 1980, 285, 66.

34. Tien, C.-J.; Sigee, D.C.; White, K.N. Characterization of surface sugars on algal cells with fluorescein isothiocyanate-conjugated lectins. Photoplasma 2005, 225, 225-233.

35. Markell, D.A.; Trench, R.K.; Iglesiasprieto, R. Macromolecules associated with the cell-walls of symbiotic dinoflagellates. Symbiosis 1992, 12, 19-31.

36. Abrego, D.; van Oppen, M.J.H.; Willis, B.L. Highly infectious symbiont dominates initial uptake in coral juveniles. Mol. Ecol. 2009, 18, 3518-3531.

37. Apprill, A.M.; Gates, R.D. Recognizing diversity in coral symbiotic dinoflagellate communities. Mol. Ecol. 2007, 16, 1127-1134.

38. Baker, A. Flexibility and specificity in coral-algal symbiosis: Diversity, ecology, and biogeography of Symbiodinium. Annu. Rev. Ecol. Evol. Syst. 2003, 34, 661-689.

39. Coffroth, M.A.; Santos, S.R. Genetic diversity of symbiotic dinoflagellates in the genus Symbiodinium. Protist 2005, 156, 19-34.

40. Pochon, X.; Gates, R.D. A new Symbiodinium clade (Dinophyceae) from soritid foraminifera in Hawai'i. Mol. Phylogenet. Evol. 2010, 56, 492-497.

41. Littman, R.A.; van Oppen, M.J.H.; Willis, B.L. Methods for sampling free-living Symbiodinium (zooxanthellae) and their distribution and abundance at Lizard Island (Great Barrier Reef). J. Exp. Mar. Biol. Ecol. 2008, 364, 48-53.

42. Adams, L.M.; Cumbo, V.R.; Takabayashi, M. Exposure to sediment enhances primary acquisition of Symbiodinium by asymbiotic coral larvae. Mar. Ecol. Prog. Ser. 2009, 377, 149-156.

43. Baird, A.H.; Bhagooli, R.; Nonaka, M.; Yakovleva, I.; Yamamoto, H.H.; Hidaka, M.; Yamasaki, H. Environmental Controls on the Establishment and Development of Algal Symbiosis in Corals. In Proceedings of the 11th International Coral Reef Symposium, Ft. Lauderdale, FL, USA, 7-11 July 2008; pp. 108-112.

44. Gomez-Cabrera, M.D.; Ortiz, J.C.; Loh, W.K.W.; Ward, S.; Hoegh-Guldberg, O. Acquisition of symbiotic dinoflagellates (Symbiodinium) by juveniles of the coral Acropora longicyathus. Coral Reefs 2008, 27, 219-226. 
45. Schwarz, J.A.; Krupp, D.A.; Weis, V.M. Late larval development and onset of symbiosis in the scleractinian coral Fungia scutaria. Biol. Bull. 1999, 196, 70-79.

46. Weis, V.M.; Reynolds, W.S.; de Boer, M.D.; Krupp, D.A. Host-symbiont specificity during onset of symbiosis between the dinoflagellates Symbodinium spp and planula larvae of the scleractinian coral Fungia scutaria. Coral Reefs 2001, 20, 301-308.

47. Abrego, D.; van Oppen, M.J.H.; Willis, B.L. Onset of algal endosymbiont specificity varies among closely related species of Acropora corals during early ontogeny. Mol. Ecol. 2009, 18, 3532-3543.

48. Chen, C.A.; Yang, Y.-W.; Wei, N.V.; Tsai, W.-S.; Fang, L.-S. Symbiont diversity in scleractinian corals from tropical reefs and subtropical non-reef communities in Taiwan. Coral Reefs 2005, 24, $11-22$.

49. LaJeunesse, T.C.; Bhagooli, R.; Hidaka, M.; DeVantier, L.; Done, T.; Schmidt, G.W.; Fitt, W.K.; Hoegh-Guldberg, O. Closely related Symbiodinium spp. differ in relative dominance in coral reef host communities across environmental, latitudinal and biogeographic gradients. Mar. Ecol. Prog. Ser. 2004, 284, 147-161.

50. Ulstrup, K.E.; van Oppen, M.J.H. Geographic and habitat partitioning of genetically distinct zooxanthellae (Symbiodinium) in Acropora corals on the Great Barrier Reef. Mol. Ecol. 2003, 12, 3477-3484.

51. Van Oppen, M.J.H.; Palstra, F.P.; Piquet, A.M.T.; Miller, D.J. Patterns of coral-dinoflagellate associations in Acropora: Significance of local availability and physiology of Symbiodinium strains and host-symbiont selectivity. Proc.Biol. Sci. 2001, 268, 1759-1767.

52. Fabricius, K.E.; Mieog, J.C.; Colin, P.L.; Idip, D.; van Oppen, M.J.H. Identity and diversity of coral endosymbionts (zooxanthellae) from three Palauan reefs with contrasting bleaching, temperature and shading histories. Mol. Ecol. 2004, 13, 2445-2458.

53. Morera, C.; Villanueva, M.A. Heat treatment and viability assessment by Evans blue in cultured Symbiodinium kawagutii cells. World J. Microbiol. Biotechnol. 2009, 25, 1125-1128.

54. Zimmermann, T.; Rietdorf, J.; Pepperkok, R. Spectral imaging and its applications in live cell microscopy. FEBS Lett. 2003, 546, 87-92.

55. Manly, B.F.J. Randomization, Bootstrap and Monte Carlo Methods in Biology, 3rd ed.; Chapman and Hall/CRC: Boca Raton, FL, USA, 2007; 1-413.

56. Pesarin, F. Multivariate Permutation Tests; John Wiley \& Sons Inc: Chichester,UK, 2001; 1-408.

57. Matlab 7.8.0.347. Mathworks: Natick, MA, USA, 2009.

58. Hayter, A.J. A proof of the conjecture that the Tukey-Kramer miltiple comparisons procedaure is conservatyive. Ann. Stat. 1984, 12, 61-75.

59. Tukey, J.W. The Problem of Multiple Comparisons; Princeton University: Princeton, NJ, USA, 1953.

60. Rodriguez-Lanetty, M.; Wood-Charlson, E.M.; Hollingsworth, L.L.; Krupp, D.A.; Weis, V.M. Temporal and spatial infection dynamics indicate recognition events in the early hours of a dinoflagellate/coral symbiosis. Mar. Biol. 2006, 149, 713-719.

61. Rodriguez-Lanetty, M.; Krupp, D.A.; Weis, V.M. Distinct ITS types of Symbiodinium in Clade C correlate with cnidarian/dinoflagellate specificity during onset of symbiosis. Mar. Ecol. Prog. Ser. 2004, 275, 97-102. 
62. Knowlton, N.; Rohwer, F. Multispecies microbial mutualisms on coral reefs: The host as a habitat. Am. Nat. 2003, 162, S51-S62.

63. Cumbo, V.R. The Establishment and Development of Symbiosis in Coral Larvae; James Cook University: Townsville, Australia, 2011.

64. Lopez-Rodas, V.; Costas, E. Characterization of morphospecies and strains of microcystis (cyanobacteria) from natural populations and laboratory clones using cell probes (lectins and antibodies). J. Phycol. 1997, 33, 446-454.

65. Koike, K.; Jimbo, M.; Sakai, R.; Kaeriyama, M.; Muramoto, K.; Ogata, T.; Maruyama, T.; Kamiya, H. Octocoral chemical signaling selects and controls dinoflagellate symbionts. Biol. Bull. 2004, 207, 80-86.

66. McAuley, P.J.; Smith, D.C. The green hydra symbiosis. V. Stages in the intracellular recognition of algal symbionts by digestive cells. Proc.Biol. Sci. 1982, 216, 7-23.

67. Voolstra, C.; Schwarz, J.A.; Schnetzer, J.; Sunagawa, S.; Desalvo, M.K.; Szmant, A.M.; Coffroth, M.A.; Medina, M. The host transcriptome remains unaltered during the establishment of coral-algal symbioses. Mol. Ecol. 2009, 18, 1823-1833.

68. Rodriguez-Lanetty, M.; Phillips, W.S.; Weis, V.M. Transcriptome analysis of a cnidarian-dinoflagellate mutualism reveals complex modulation of host gene expression. BMC Genomics 2006, 7:1-7:23.

(C) 2011 by the authors; licensee MDPI, Basel, Switzerland. This article is an open access article distributed under the terms and conditions of the Creative Commons Attribution license (http://creativecommons.org/licenses/by/3.0/). 